\title{
COVID-19 testing and cases - a complex relationship in three acts.
}

\author{
Claus Heinrich
}

\begin{abstract}
If and how the increasing numbers in confirmed COVID 19 cases are driven by the simultaneous expansion of test capacities and tests conducted is a question under continuous debate. Building on the hypothesis, that the impact of increased testing might not be constant over time but determined by the national test policy applied, the relationship between testing and number of cases was investigated in different phases of the pandemic with a focus on the time before and after a change in German legislation on May 22, 2020 that opened the door for more tests in asymptomatic persons for prevention and containment purposes. Based on linear regression models it is estimated that about $60 \%$ of the cases tested positive after May 22 can be attributed to increased testing, while eliminating the test effect does not substantially impact the numbers before that date. Likewise, the clinical presentation of cases registered after May 22 is significantly different, with a hospitalization rate of $8.12 \%$ (before $18.17 \%$ ) and a case fatality rate of $0.63 \%$ (before $5.17 \%$ ).

It is concluded that expanding the number of tests as it was done in Germany did not lead to a better understanding of the epidemiology of COVID 19. It is recommended to separate test results from preventive testing and testing based on a clinically defined test strategy, and to only use the latter for surveillance and as a basis for political decisions.
\end{abstract}

\section{Introduction}

Germany, like many other countries, is currently facing an increase of confirmed SARS-COV- 2 infections detected by PCR-tests and daily reported by the German institute for disease control ("Robert Koch Institut" - RKI). However, the rate of severe cases with hospitalizations and the case fatality rate remain on a stable low level and the number of tests conducted was significantly increased since end of May. While it is generally acknowledged, that the amount of testing does play a role, there is a clear dissensus and increasingly emotional debate about the extent. Opinions vary from completely denying any new infections, stating that all test-positive cases would be false-positive, to completely denying the relevance of the question, assuming that each positive PCR test constitutes a danger to society and needs to be avoided at all cost.

In my previous paper "Hunting the Tiger" [1] I have already shown that neither the number of tests conducted alone, nor the measures taken in response to the outbreak show a convincing causal relation to the daily number of confirmed infections. I had stated as a hypothesis, that the test strategy applied could play a more important role than the absolute number of tests conducted. The German ministry of health changed legislation with effect of May 22, 2020 [2] to allow for more tests in asymptomatic persons, leading to a continuous increase in the number of individuals tested since then. Tests were conducted in selected groups rather than aiming for representativity, like returning vacationers or employees of factories, where individual infections had been detected. Before that date mainly patients with symptoms, who had been in contact with a confirmed case, were tested, with some clearly restricted exceptions like people working in the health sector. I am using this occasion as a natural experiment to further explore the interaction between test strategy, number of tests, and number of confirmed cases in the following. 


\section{Objectives}

- To analyze and describe how strong the number of registered SARS-COV-2 infections is determined by national test strategy and extent of testing

- To assess and quantify the impact of changing the national test strategy on May 22 on the number of SARS-COV- 2 infections registered since that date

\section{Data}

This manuscript is based on daily reports of confirmed COVID 19 cases in Germany using the dataset available on https://npgeo-corona-npgeo-de.hub.arcgis.com. The data analyzed cover a timeframe from 02. March to 06 Sep. 2020. Due to the daily updates from local health authorities the numbers are sometimes adjusted retrospectively. My dataset represents the status of 11 Sep. 2020. The "disease onset date" variable contained in this dataset was used for aggregation of case numbers, which is thought to be less affected by administrative reporting delays than the "report date". Only where no clinical information on date of symptom onset is available, the RKI substitutes by the date of report.

Information on PCR test activities per calendar week was derived from the regular publications issued by the RKI on that topic within the institutes regular journal "Epidemiological Bulletin". Every Wednesday, cumulative data on test activities are presented, with a break-out by calendar week starting from week 11 and summarizing all reported tests up to week 10. The numbers were extracted from edition 37/2020 [3]. Data for calendar week 10 were included with half of the tests recorded up to that week in absence of a more precise approximation.

Some basic clinical data are reported with the regular RKI situation report on COVID 19, differentiated by calendar week and for cases recorded during a week. The number of patients with symptoms, hospitalization and fatal outcome for the period discussed in this paper was derived from the RKI situation report published on October 06, 2020 [4].

\section{Methods and results}

\section{Global interaction model}

Building on considerations outlined in "Hunting the Tiger" [1] it is probably not adequate to view the curve of daily confirmed infections as a homogenous development. The curve is rather driven by varying constellations of actual disease proceedings, test strategy, and individual political decisions in distinct situations. For the purpose of this analysis I am differentiating between three phases with potentially different interactions between test strategy and underlying disease dynamics that can be described as follows:

- Phase A (calendar week 10/11): Initial exponential increase, symptom-based testing

- Phase B (calendar week 12 - 21): Linear decline, symptom-based testing

- Phase C (calendar week 22 - 36): Alternating curve with increasing trend, extended testing

Figure 1 marks these phases in a graph showing the moving average of daily RKI-confirmed cases on a calendar week basis. The cutting point between Phase $B$ and $C$ is the date when revised law on infection protection took effect which allowed for extended testing in asymptomatic persons [2]. 


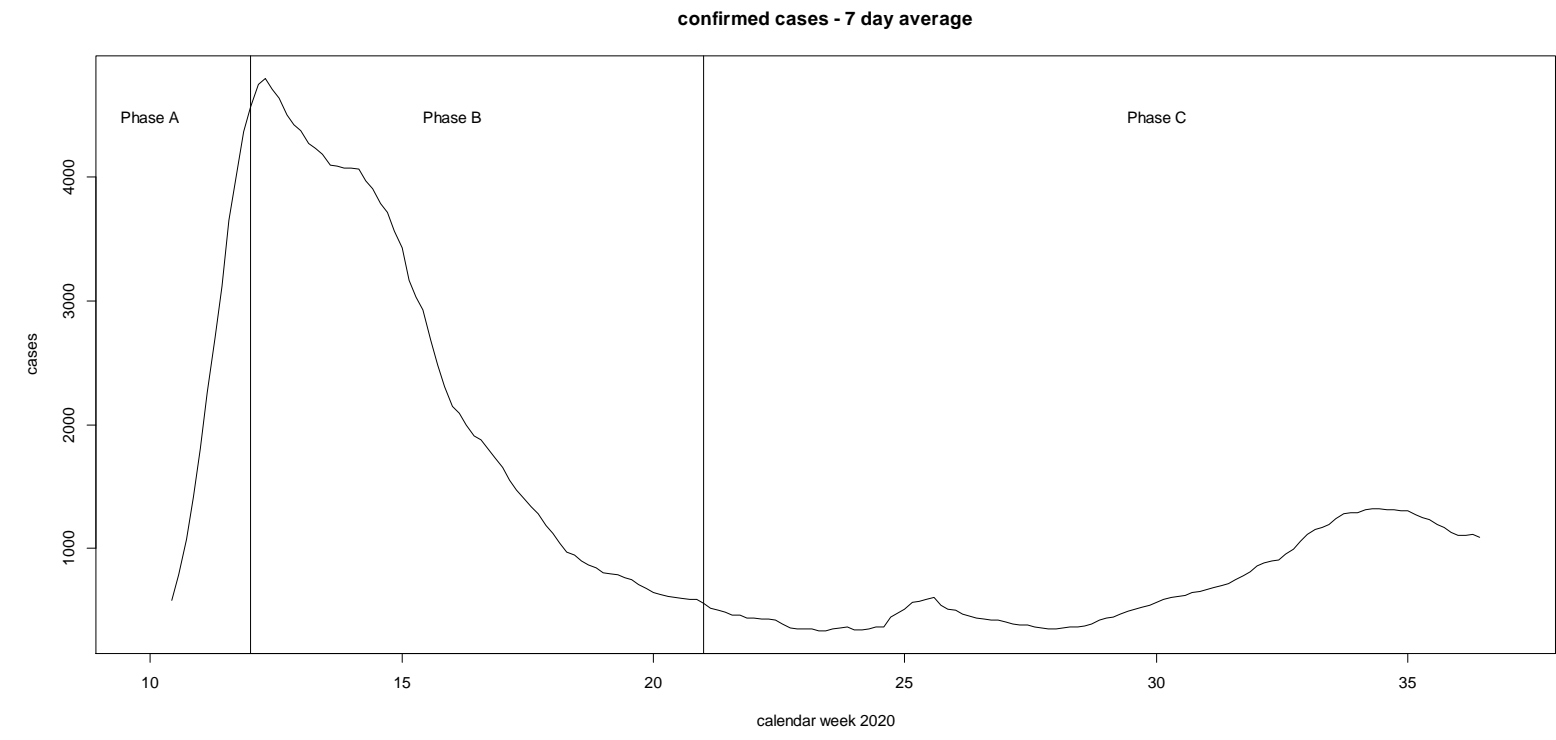

Figure 1: Phases of the Pandemic in Germany

In my previous paper I have found a statistically significant, yet numerically weak impact of testing on number of registered infections when used as a predictor variable in a linear model and only considering Phase A and B. One possible explanation for this finding could be that those external factors I have used to distinguish the phases influence the nature of relationship between testing and positive findings, thus compromising linearity. Phase $\mathrm{B}$ and $\mathrm{C}$ might differ because the test strategy has been changed. Phase A has a clearly distinct characteristic and might be dominated by what I have called a "calibration artifact" in "Hunting the Tiger" [1]. A linear regression model with interaction term was used to test these general considerations.

Combining phase and testing as factorial and linear predictors in an interaction model for the number of confirmed cases yields the following coefficients:

Coefficients:

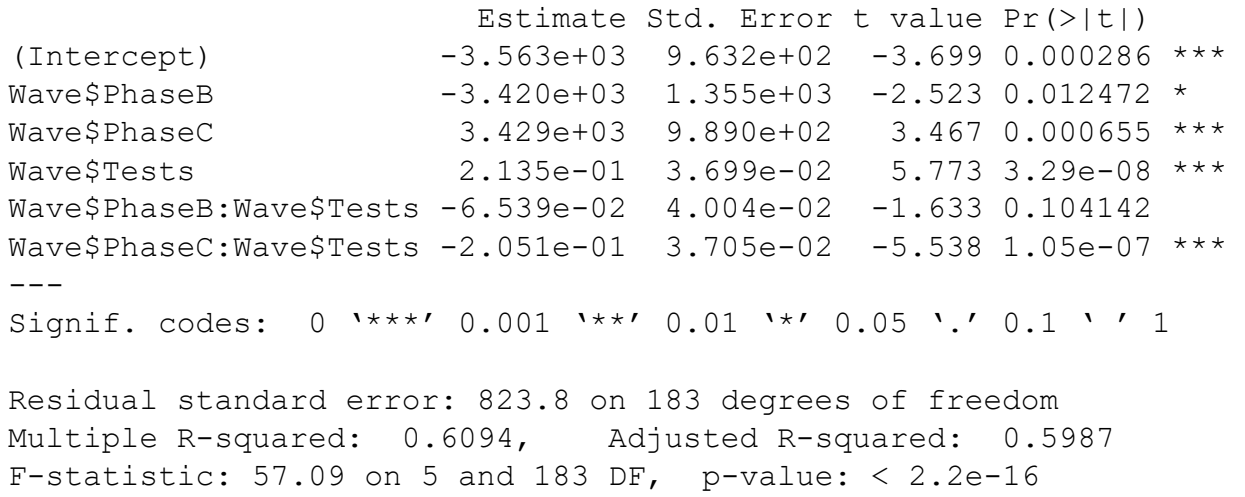

With an R-squared of .61 $\left(p<2.2^{-16}\right)$ the model has a strong predictive value and demonstrates significant contributions for the factorial predictor (phase), the numerical predictor (tests), as well as the second interaction term. An analysis of variance shows the main effects as well as the interaction effect highly significant with the "phase"-factor and the interaction term "phase x tests" explaining the majority of observed variance: 


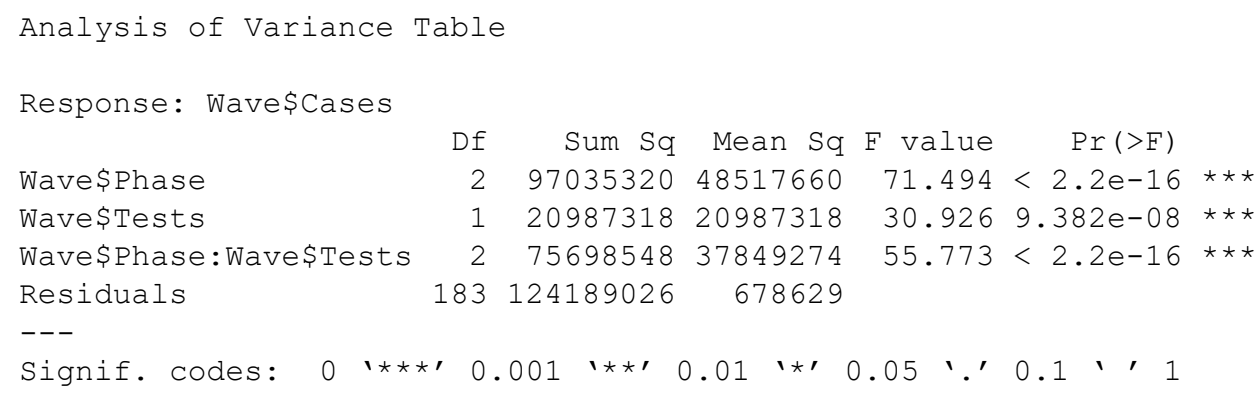

The results as described above clearly support the idea that the relation between testing and rate of identified cases is altered by external circumstances in different phases of the pandemic. This affects the strength of correlation as displayed in Table 1.

Table 1: Correlation coefficients tests/cases by phase

\begin{tabular}{|r|r|r|r|r|}
\hline & $\begin{array}{r}\text { Correlation } \\
\text { (Pearson) }\end{array}$ & t-value & $\begin{array}{r}\text { Degrees of } \\
\text { freedom }\end{array}$ & p-value \\
\hline Phase A & 0.9005887 & 7.1772 & 12 & $1.121 \mathrm{e}-05$ \\
\hline Phase B & 0.5976083 & 6.1463 & 68 & $4.712 \mathrm{e}-08$ \\
\hline Phase C & 0.8312878 & 15.178 & 103 & $<2.2 \mathrm{e}-16$ \\
\hline
\end{tabular}

Unfortunately, testing data on phase A (initial increase) are insufficient to allow for any further analyses in this direction. However, comparing the data before and after the change of national test policy on 22 May could open the door to a better understanding how this change impacted what we typically view as the daily rate of new infections. In the following we will have closer look at Phase B and $C$ individually.

\section{Phase B - linear decline}

As it can be seen from Figure 1, the period between March 16 and May 22 ("Phase B") shows an almost linear decline of daily confirmed cases. At the same time there is still a substantial correlation with background test activity (compare Table 1). For further clarification, a linear model was constructed for Phase B, including the number of tests as a predictor. To address the visible time-trend the number of calendar days was included as a second (surrogate) predictor. The model was constructed without interaction term and yielded the following coefficients:

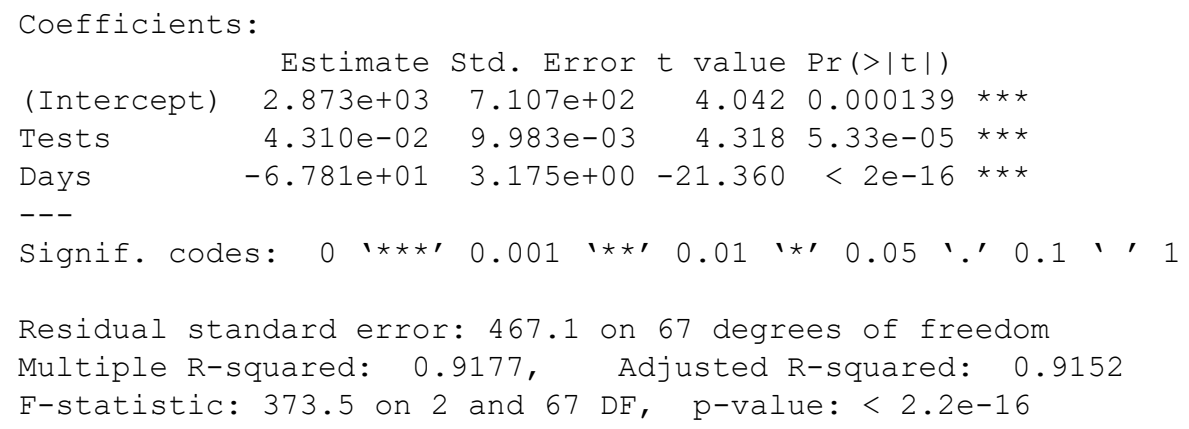

With an adjusted $\mathrm{R}$-squared of $0.9152\left(\mathrm{p}<2.2^{-16}\right)$ the model explains most of the observed variance of confirmed cases over time. An ANOVA shows that the test effect accounts for about $35 \%$ of explained variance in this model, $56 \%$ is explained by the (decreasing) time trend, i.e. the number of days passed: 


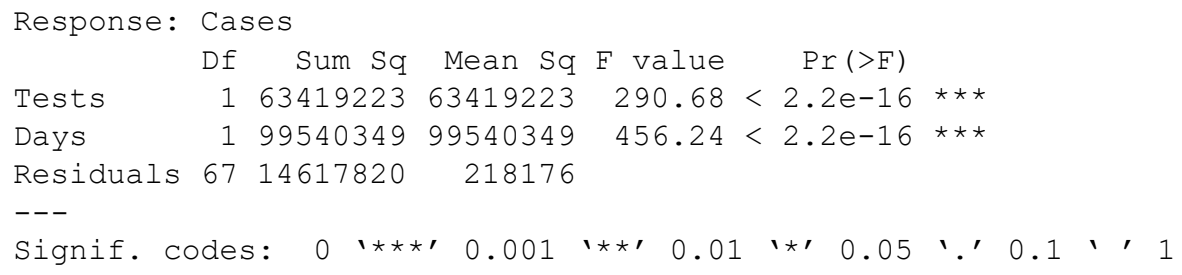

Does the amount of testing impact the absolute number of positive results? To address this question, a further linear regression model was calculated predicting number of confirmed cases only from the number of tests. The residuals obtained from this model represent the development after elimination of the test effect, yet at a numerically lower level. To make them comparable to the originally observed values a linear transformation was performed shifting the minimum value from negative to zero, thus pushing the curve up the $y$-axis. Figure 2 shows how the transformed residuals (dotted line) overlap with the originally observed values (solid line). The graph shows the residuals in an irregular zigzag across the original curve but eliminating the test effect does not seem to alter the general downward trend. Summing up both variables and setting them into relation gives a ratio of 1.017 for transformed residuals to observed, meaning that the total number of cases is almost the same.

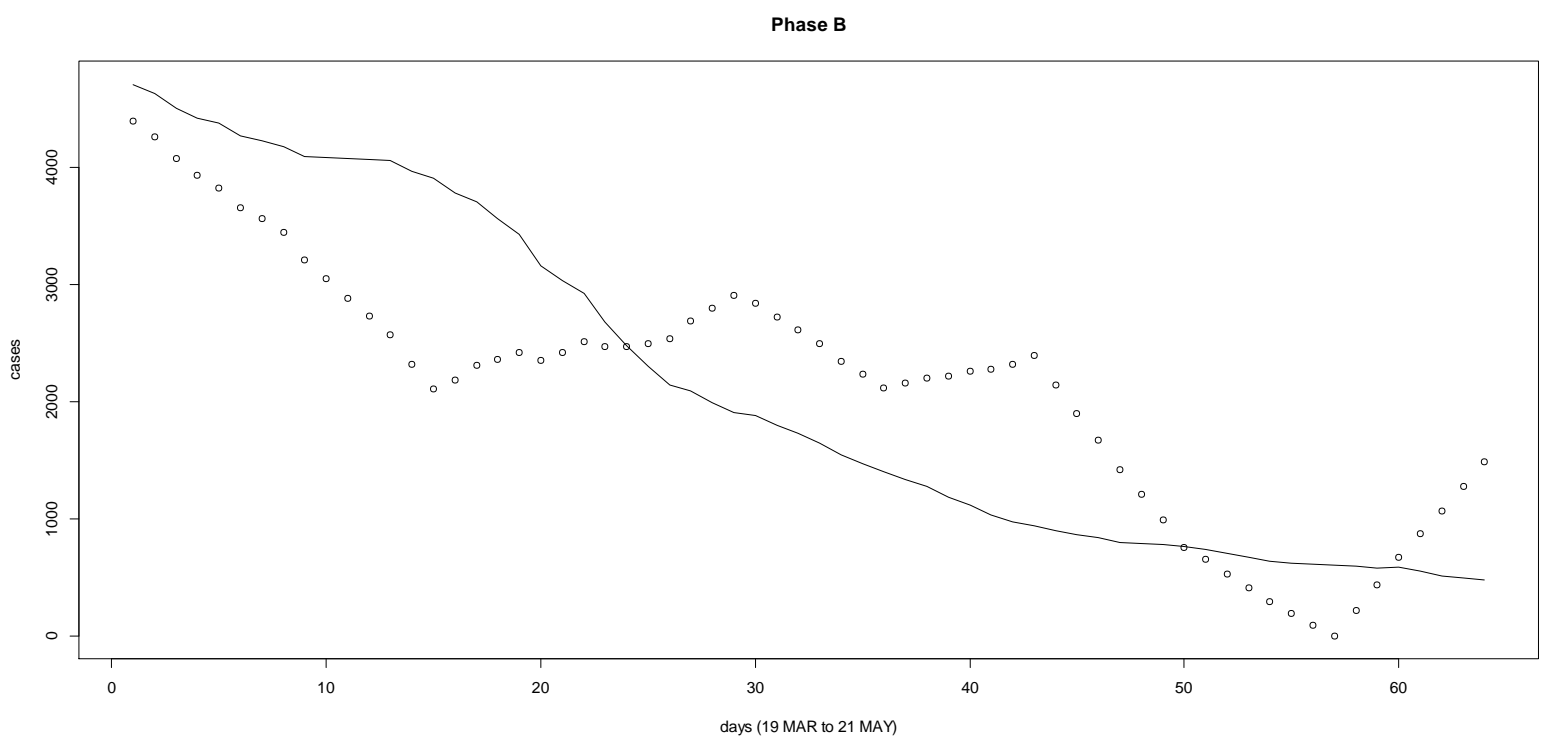

Figure 2: Reported cases (solid line) and shifted residuals (dotted line) - Phase B

\section{Phase $\mathrm{C}$ - extended testing}

The same set of analyses was repeated for Phase C. The regression model including time trend and test activities yielded the following coefficients:

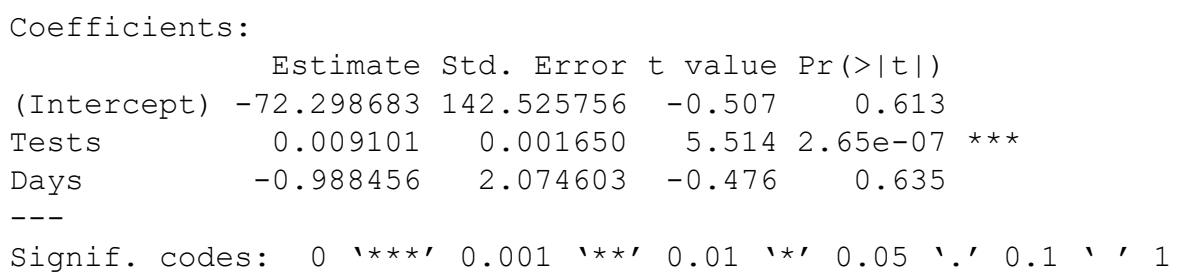

The model shows a significant effect of the test-variable only, but again has a high predictive value with about $69 \%$ explained variance. An ANOVA does not yield any additional information in this case, since only the number of tests has a significant impact on the dependent variable. As described for 
Phase $B$, a second model was constructed, and the residuals extracted to eliminate the test effect. Figure 3 plots the shifted residuals (dotted line, compare previous section) against observed cases as reported by the RKI (solid line). This gives a completely different picture compared to phase B (Figure 2). Starting at similar levels there is an increasing gap between the two curves over time while the residuals show a sort of sine wave around a constant level of about 300 cases, yet without an obvious trend in any direction. The observable global upwards trend in the RKI-curve seems to be completely driven by the simultaneous increase in test activities. Here, the sum of shifted residuals is only $40 \%$ of summed up observed cases. It is also illustrative to look at the relation between reported and testcorrected case numbers at individual timepoints. While these are pretty close in the beginning of Phase C, towards the end reported numbers are about eight to ten times higher than the test-corrected values.

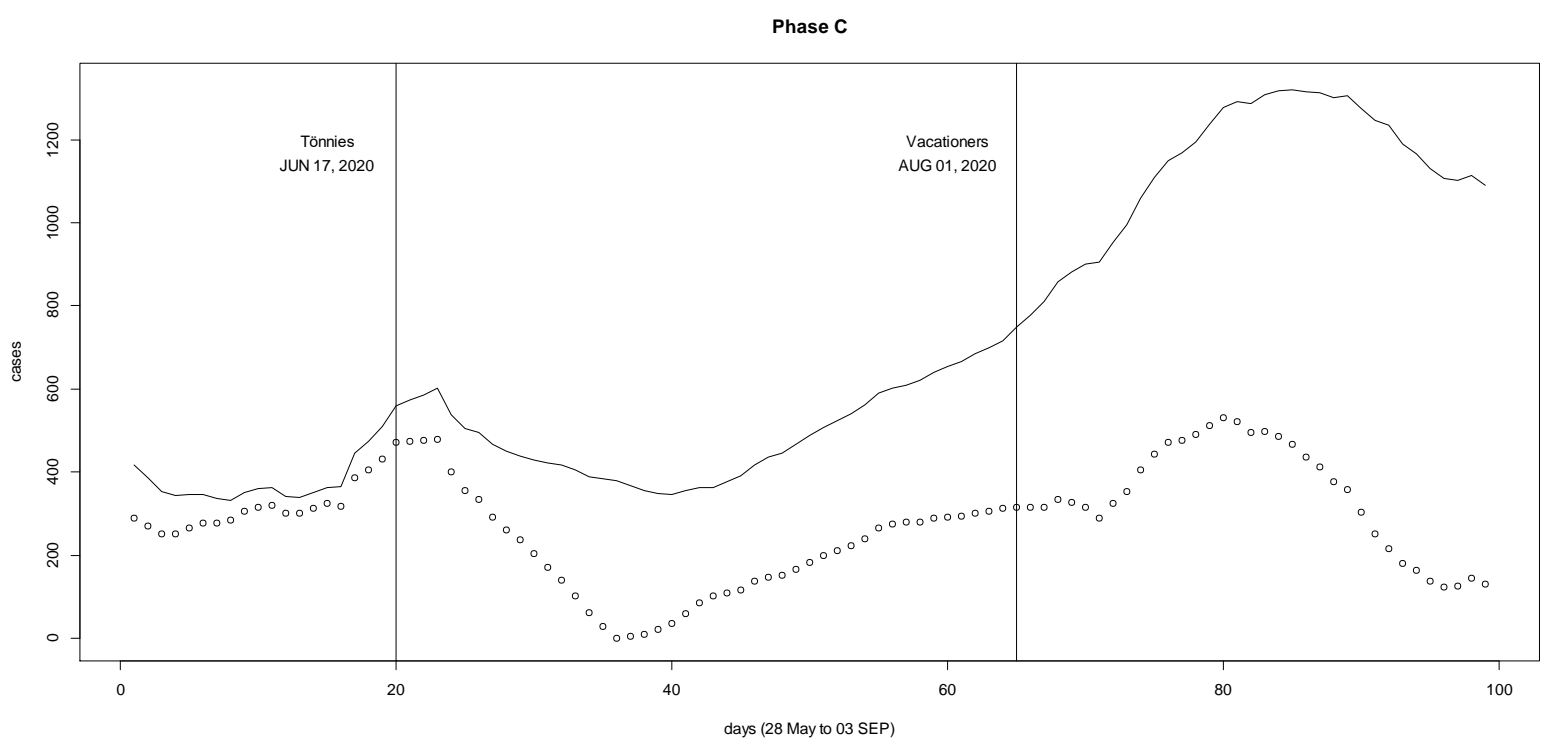

Figure 3: Reported cases (solid line) and shifted residuals (dotted line) - Phase C; time markers indicate the "Tönnies"outbreak and the date when Germany started offering free tests to all vacationers returning from abroad (see discussion)

\section{Clinical information}

As I have already stated above, the development in terms of hospitalization and death related to COVID 19 is far less alarming than it might be expected from the increasing number of cases recorded when compared to the situation in early spring. Table 2 compares clinical information for the three phases based on numbers reported by the RKI.

Table 2: Clinical information by phase

\begin{tabular}{|l|r|r|r|r|}
\hline & average age & symptomatic (\%) & hospitalized (\%) & deaths (\%) \\
\hline Phase A & 43.76 & 94.50 & 10.64 & 1.32 \\
\hline Phase B & 49.10 & 91.78 & 18.17 & 5.17 \\
\hline Phase C & 34.48 & 71.60 & 8.12 & 0.63 \\
\hline
\end{tabular}

Not surprisingly, the clinical presentation of cases identified in Phase $\mathrm{C}$ is dramatically different from Phase $A$ and $B$. The test strategy applied does not only affect the number but also the typical clinical presentation of cases registered as new infections by public surveillance. 


\section{Discussion}

The results as presented above impressively confirm the initial hypothesis that external factors, like a change of test strategy, heavily impact the way how the number of tests conducted correlates with the number of cases detected. For the phase of steady decline in daily numbers from mid-March to mid-May (Phase B) we see a sort of balance between tests and cases. This kind of relationship can be expected if two variables are mutually dependent. The correlation is mainly driven by the underlying mechanism that new cases are predominantly identified by looking for symptomatic contacts of already confirmed cases. This way cases beget tests beget new cases, while the overall trend is dominated by epidemiological development. The continuous decline of infections could for example be a seasonal effect as it is typically seen for respiratory virus. Controlling the impact of varying test activity does not change how many cases are detected, since the number of tests performed follows the number of cases and the other way round. During Phase B the daily surveillance system probably gave a pretty accurate picture of infection proceedings in the general population, even though it showed only a small segment of the true infection rate with a considerable number of unreported cases.

The picture completely changes after May 22, when the regulations in Germany were adapted to allow for arbitrary testing for prevention and containment purposes (Phase $\mathrm{C}$ ). At this time, the overall population trend had already reached its' low and would have idled around some 200 to 300 infections per day if testing would not have been expanded. Instead, there was a first test to the new regulation with the "Tönnies" case, a major outbreak event in a meat factory in Northern Rhine area. Following a high rate of positive test results in one factory area, the whole company staff of some 7000 employees was tested, yielding a total of more than 2000 confirmed cases. For whatever reason, this incident seems to have caught a lot less scientific attention than famous cruising ship events like the Diamond Princess outbreak and I am really wondering why. Only recently at least a journalist reported some basic numbers via a German news portal as she had learned them during an interview with the local health authority [5]. There are at least two remarkable aspects in these numbers which would have deserved a more thorough investigation:

1. Only less than one third of the employees caught the virus under allegedly worst possible conditions in terms of infection prevention. The employees, who were mainly coming from other EU countries and remained isolated from the local population, were in close contact throughout the day at work without any protection and were crowding within their housing blocks after they were quarantined, as it could be seen in the news day by day. I think this was a great opportunity missed to find out more about contagiousness and protective factors if there would have been any interest in this.

2. Of 2,119 persons tested positive, 41 were eventually hospitalized. It is not clear how many required ventilation - if any - but it is reported that none of them died, so we have a hospitalization rate of $1.9 \%$ and a fatality rate of $0 \%$ in this group. The author of the article mentions the probably "robust health" of the workers as the most plausible explanation for these unexpected mild consequences, yet I guess this is a rather romantic picture of people from poor countries migrating to Germany for hard work under miserable conditions and isolated from their families. I for my part would rather see a high chance for stress, pain and sleep medications, antidepressants, unhealthy diet, alcohol, and nicotine helping survive. In other words, this would be a promising group to look for the rare COVID 19 risk patients among the young.

After the Tönnies incident the decision to offer free testing for returning vacationers upon return led to the next peak of the curve. However, the results as presented above show that expanding the tests 
the way it was done in Germany did not lead to a better understanding of the epidemiology of this disease as compared to the situation before, rather the contrary:

- About $60 \%$ of the newly detected infections were causally attributed to the increased number of tests, without conveying information on population developments.

- The new test strategy identified a population that exhibits a rather low risk to develop severe symptoms and is probably not substantially driving the pandemic or putting the health system at risk.

- The targets for expanded testing were not chosen for representativity but driven by preventive intentions, like reducing the risk of new infections brought in from abroad. Still all positive findings were summed up to an increasingly meaningless "total of infections" without any differentiation.

Maybe accurate monitoring of the epidemiological situation was not the core priority behind expanding test capacities, yet to this respect the outcome still is a complete disaster. By increasing the number of tests and choosing groups for expanded asymptomatic testing without any clinical rationale we gave away the pretty good view on the epidemiological situation we had before May 22. What we did was like tuning a transistor radio away from the radio station frequency and simultaneously amplifying the volume ten times. What we got in return was amplified white noise, which makes it very difficult now to understand how dangerous the undoubtedly rising numbers really are, that concern us so much since September.

Until May 22, Germany conducted about one third of the tests compared to the situation today. Now more than one million tests are conducted each week, lab supplies are increasingly scarce and laboratories at the verge of collapse. The majority of these tests are wasted to asymptomatic people for various reasons, yet without any true benefit for improving surveillance or containing infections. A highly sensitive test method developed for supporting or confirming a clinical diagnosis is misused as a screening method, with more and more people labelled as infected without any follow up information on symptomatology and severity of disease. At the same time there are increasing concerns that supplies could be short for areas, where extensive preventive testing would really make sense, like nursing homes or hospitals.

This unfortunate situation is deteriorated by ever increasing efforts to trace back each and every contact of each and every test-positive person, regardless if the contacts identified are at increased risk or not. The majority of these ever-growing efforts yields asymptomatic or mildly symptomatic cases infecting further mildly or asymptomatic cases that would probably not require hospitalization anyway. Still these activities will foreseeably block and finally overload the system to degree that will force a decision to go into the next lockdown or just give up the struggle.

There are some possible steps that would help to quickly re-gain control over the situation and help steering any required measures more effectively:

1. Tests conducted in asymptomatic persons for preventive purposes, like those performed in returning vacationers, should be kept apart from the numbers used for epidemiological surveillance. Cases used for epidemiological surveillance should be restricted to cases identified according to a clinically meaningful test strategy. A good example how this can be done was given by Sweden back in June, when they moved from testing cases with symptoms and confirmed contact to an infected to testing all persons with respiratory symptoms. This led to a sudden and sharp increase in numbers, but the curve quickly adapted to the new strategy and started to decline again. They expanded their testing but still maintained a good overview on their epidemiology this way. 
2. Preventive testing in asymptomatic or mildly symptomatic persons should be strictly reserved for people at increased risk - mainly the elderly - and people frequently in close contact with high risk persons, like nurses or care-giving family members.

3. Low-risk contact persons of confirmed COVID 19 cases should not be tested unless they really get seriously ill. They should be reminded to stay at home when they feel even slight respiratory symptoms, otherwise keep distance to potential risk groups and avoid group events for one or two weeks. That would mean to work from home where possible, avoid visiting aged relatives and postpone the birthday party, yet it would probably not be necessary to lock them away completely.

If we would move to these simple steps I am sure we would immediately see the numbers go down, have a better understanding of the epidemiological situation, significantly reduce the workload for contact tracers, have better protection for risk groups, and still would have the option to return to stronger measures in case we would see an alarming increase of severe cases. Still, whatever we do the way we are currently going is leading nowhere, as we are going to be completely overwhelmed with meaningless cases before winter even starts. Individual contact tracing on each test-positive case and mass testing only makes sense with abundant capacities and if there is a reasonable chance to eradicate the virus from the population - which is not going to happen. I think it is high time to accept that and live with it.

\section{References}

[1] C. Heinrich, "Hunting the tiger - what can be learned from public surveillance data on the population risk caused by SARS-COV-2?," Advance. Preprint. https://doi.org/10.31124/advance.12922331.v1, 2020.

[2] Bundesministerium für Gesundheit (BMG), "Zweites Gesetz zum Schutz der Bevölkerung in einer epidemischen Lag von nationaler Tragweite," Bundesgesetzblatt, pp. 1018 -1036, 22 May 2020.

[3] Robert Koch Institut, "Erfassung der SARS-CoV-2-Testzahlen in Deutschland (Stand 09.09.2020).," Epid Bull 37, pp. 12-15, 2020.

[4] Robert Koch Institut, "Coronavirus Disease 2019 (COVID 19). Daily Situation Report of the Robert Koch Institute.," 06 October 2020.

[5] M. Schmude, "Was aus den Infizierten bei Tönnies wurde," https://www.deutschlandfunk.de, 01 September 2020. 